\title{
A novel wavy channel heat exchanger: the sine-helical mixer
}

\author{
Abbas Aldor ${ }^{1,2}$, Yann Moguen ${ }^{1}$, Kamal El Omari ${ }^{3,1}$, Charbel Habchi², Pierre-Henri Cocquet ${ }^{1,3}$, \\ Yves Le Guer ${ }^{1}$ \\ ${ }^{1}$ Laboratoire des Sciences de l'Ingénieur Appliquées à la Mécanique et au Génie Électrique (SIAME) / Université de Pau \\ et des Pays de l'Adour, E2S-UPPA, Pau, France \\ abbas.aldor@univ-pau.fr; yann.moguen@univ-pau.fr; pierre-henri.cocquet@univ-pau.fr; \\ ${ }^{2}$ Notre Dame UniversityCompany / Thermofluids Research Group, Zouk Mosbeh, Lebanon \\ charbel.habchi@ndu.edu.lb \\ ${ }^{3}$ Laboratoire de Physique et Ingénierie Mathématique pour l'Énergie et l'Environnement (PIMENT) / Université de la \\ Réunion, Sainte Clotilde, France \\ kamal.el-omari@univ-reunion.fr
}

\section{Extended Abstract}

Flows in curved channels are often used for many industrial operations: heat transfer, chemical reaction, filtration, separation, dispersion, etc. [1]. In such flows, due to the centripetal forces and the viscous drag along the walls, two symmetrical counter-rotating vortices, termed Dean vortices or Dean cells, can develop. In a helical channel flow, the Dean cells are modified by the torsion, which causes the plane separating the cells to rotate. The particle trajectories are then toroidal and no exchange of fluid occurs between the two vortices. However, by twisting two consecutive portions of a helical channel, the separatrix between the Dean cells can be broken. Complex trajectories and chaotic advection of particles are then obtained $[2,3,4]$. In the present study, we present an innovative geometry for a passive mixer, used here as a heat exchanger, whose channel can be seen as a superimposition of an oscillating channel on a standard helical channel. It is thus a wavy channel based on a sine-helical geometry.

In the present study, we consider a Newtonian fluid. The flow is steady, incompressible and laminar. The fluid temperature at the wall is chosen with a certain deviation from the fluid temperature at the inlet. The Prandtl number is close to that of water, $\operatorname{Pr}=7$. The Reynolds number Re ranges from 100 to 1400 . The helical and the sine-helical channel lengths are identical. Both mesh sizes are also approximately the same, with 3 e6 cells.

For typical results obtained, we observe that the flow reaches rapidly a hydrodynamically fully developed state in the case of the helical configuration. Correlatively, the thermal field reaches a thermally fully developed state, but with a longer length. This is not the case for the sine-helical configuration for which the velocity field exhibits a strong periodic modulation along the channel, which results in the creation of a thermal strange eigenmode pattern for the thermal field observed at periodic locations along the channel. Thus, the additional degree of freedom of the sine-helical geometry allows a modulation of the velocity field that makes the fluid mixing more efficient.

The potentiality of the sine-helicoidal flow has been proved to enhance thermal mixing by chaotic advection. This mathematically parameterizable geometry offers potential for the search of optimal geometries for the design of innovative and highly efficient mixers, heat exchangers and reactors. The expectations of this study are then fulfilled: the sine-helical heat exchanger has proved its effectiveness compared to a more traditional solution commonly used in the industry, the helical heat exchanger.

\section{References}

[1] S. Vashisth, V. Kumar and K.D.P. Nigam, A review on the potential applications of curved geometries in process industry. Ind. Eng. Chem. Res. 47: 3291-3337, 2008.

[2] S.W. Jones, O.M. Thomas and H. Aref, Chaotic advection by laminar flow in a twisted pipe. J. Fluid Mech. 209:335$357,1989$.

[3] Y. Le Guer and H. Peerhossaini. Order breaking in Dean flow. Phys. Fluids 3:1029-1032, 1991.

[4] S.A. Bahrani, L. Humberset, R. Osipian, L. Royon, K. Azzouz, A. Bontemps. How thermally efficient are chaotic advection mixers? An experimental assessment. Int. J. Therm. Sc., 145, 2019. 